\title{
Analysis of the Drosophila Compound Eye with Light and Electron Microscopy
}

\author{
Monalisa Mishra and Elisabeth Knust
}

\begin{abstract}
The Drosophila compound eye is a regular structure, in which about 750 units, called ommatidia, are arranged in a highly regular pattern. Eye development proceeds in a stereotypical fashion, where epithelial cells of the eye imaginal discs are specified, recruited, and differentiated in a sequential order that leads to the highly precise structure of an adult eye. Even small perturbations, for example in signaling pathways that control proliferation, cell death, or differentiation, can impair the regular structure of the eye, which can be easily detected and analyzed. In addition, the Drosophila eye has proven to be an ideal model for studying the genetic control of neurodegeneration, since the eye is not essential for viability. Several human neurodegeneration diseases have been modeled in the fly, leading to a better understanding of the function/misfunction of the respective gene. In many cases, the genes involved and their function are conserved between flies and human. More strikingly, when ectopically expressed in the fly eye some human genes without a Drosophila counterpart can induce neurodegeneration, detectable by aberrant phototaxis, impaired electrophysiology, or defects in eye morphology. These defects are often rather subtle alteration in shape, size, or arrangement of the cells, and can be easily scored at the ultrastructural level. This chapter aims to provide an overview regarding the analysis of the retina by various means.
\end{abstract}

Key words: Drosophila melanogaster, Light microscopy, Deep pseudopupil, Electron microscopy, Cryolabeling, Whole mount, Compound eye

1. Introduction

\subsection{The Structure of the Drosophila Compound Eye}

The Drosophila compound eye is a highly ordered structure composed of about 750 functional units called ommatidia, which are regularly arranged in a hexagonal pattern, visible from the outside by the facets, which are formed by the lenses (Fig. la). Each ommatidium is an elongated, barrel-like structure of about $100 \mu \mathrm{m}$ in length, the distal $10 \%$ of which is occupied by the cornea and the crystalline cone, which together form the dioptric apparatus (Fig. 2a). Below, the eight, highly elongated photoreceptor cells (PRCs) follow, which are associated with pigment and cone cells. The cell bodies 

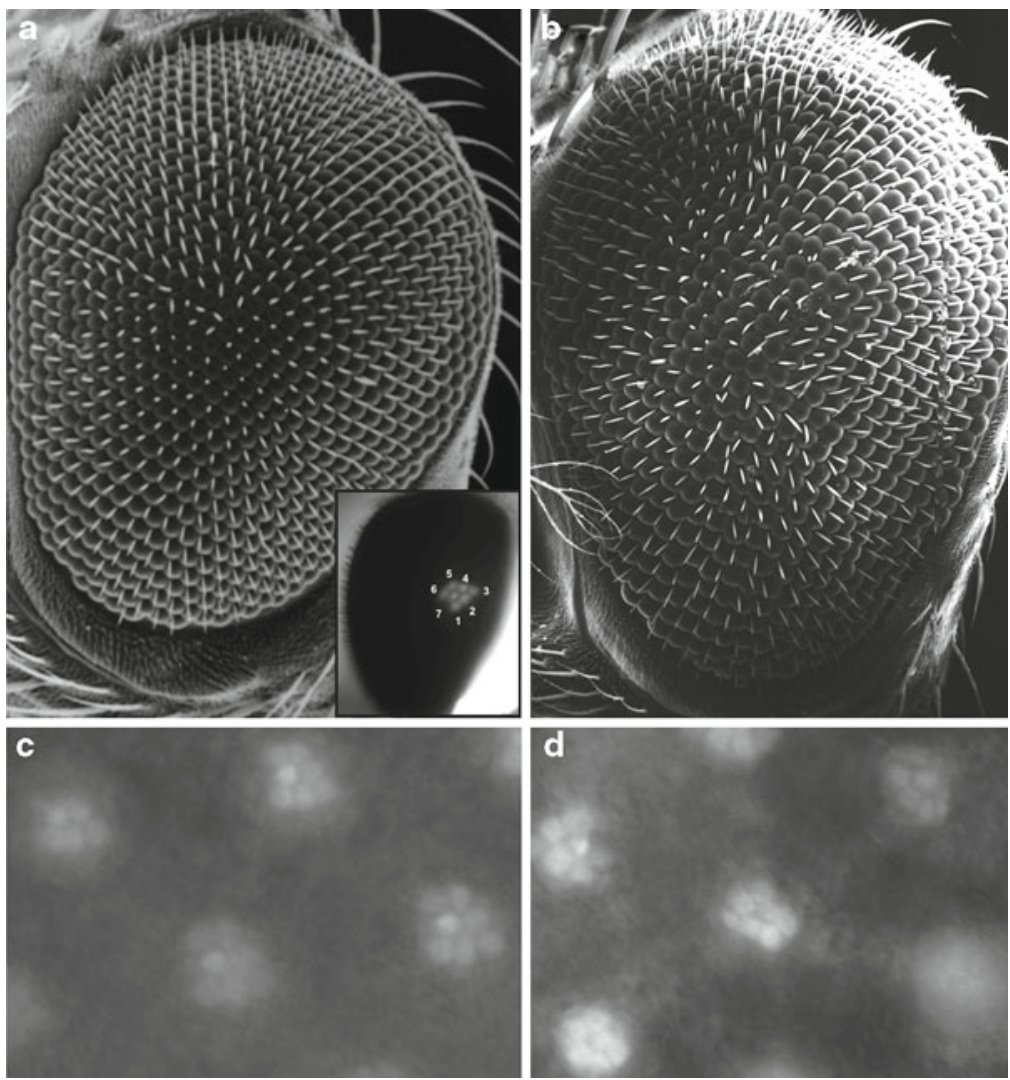

Fig. 1. Overview of the adult Drosophila eye. (a) Scanning electron micrograph (SEM) of a wild-type Drosophila compound eye showing the highly regular arrangement of facets. Each lens has a diameter of approx. $18 \mu \mathrm{m}$. Inset: Light micrograph of a wild-type eye revealing the deep pseudopupil in the form of a trapezoid rhabdom. (b) Overexpression of the cytoplasmic domain of Crumbs in all cells of the eye results in a rough eye phenotype. (c) Optical neutralization of the wild-type eye showing the trapezoid arrangement of the rhabdomeres. (d) Optical neutralization of a fly expressing crb RNAi shows the loss of the trapezoid arrangement of the rhabdomeres (c and $\mathbf{d}$ kindly provided by N. Gurudev).

of the outer PRCs Rl-R6 span the entire length of the retina and project axons to the lamina, the first optic neuropile. The axons of the central R7 and R8 cell synapse in the medulla, the second neuropile of the optic lobe.

All eight PRCs of an individual ommatidium point their apical membrane towards the center (Fig. 2c). The apical membrane itself is subdivided into the most apical rhabdomere and the stalk, which connects the rhabdomere with the zonula adherens (ZA), and which corresponds to the inner segment of the vertebrate PRC (Fig. 2d). The rhabdomere, the light-sensing organelle, is composed of an array of densely packed microvilli of $1.5 \mu \mathrm{m}$ in length and about $60 \mathrm{~nm}$ in width, which accounts for about $90 \%$ of the cell's plasma membrane and harbors the visual pigment rhodopsin. The eight rhabdomeres within each ommatidium are arranged in a 

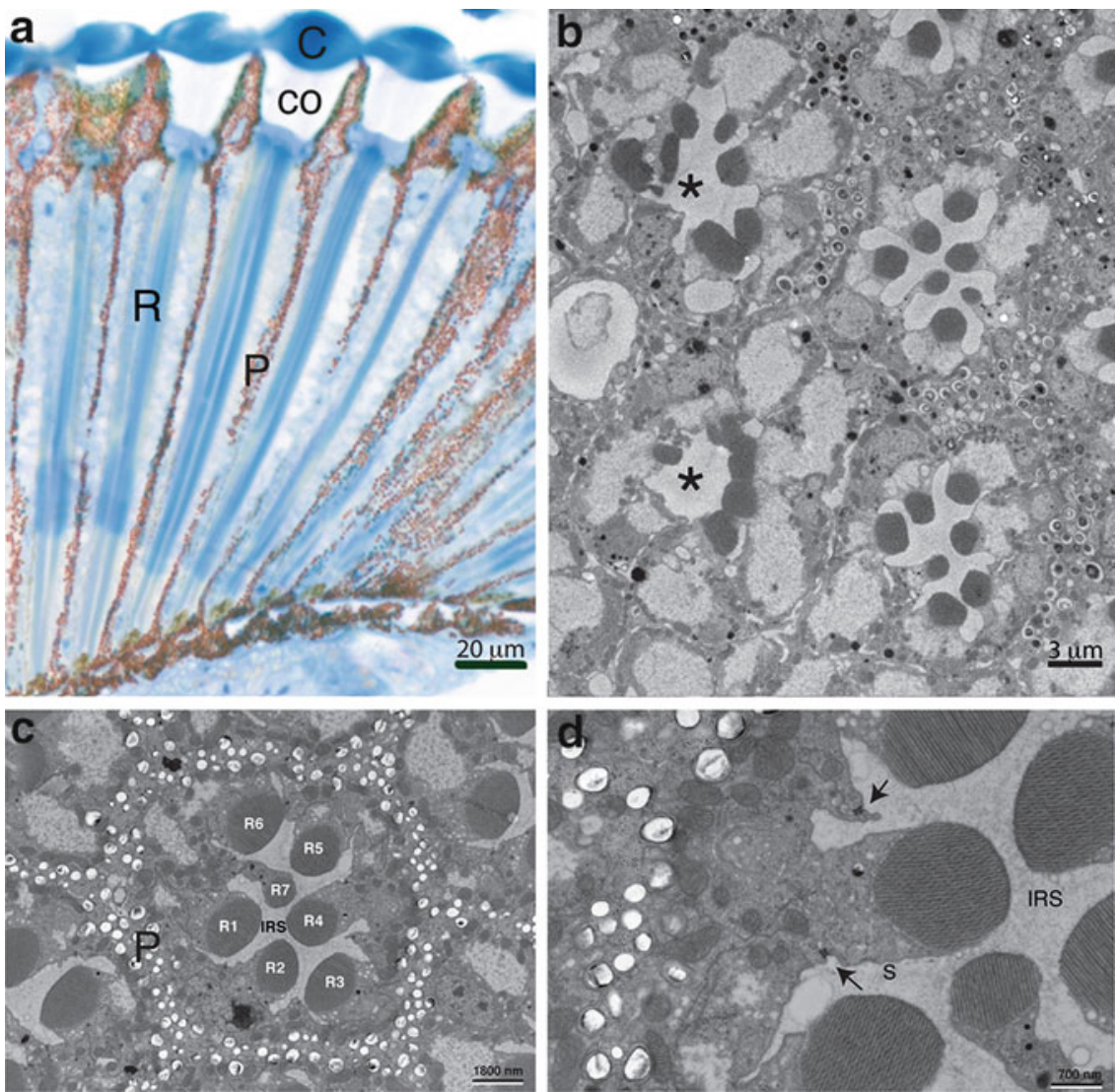

Fig. 2. General organization of the compound eye. (a) Light micrograph of a longitudinal section illustrating the cornea (C), the cone (co), the rhabdom (R), and the pigment in the pigment cells $(\mathrm{P})$. Note that pigment cells separate ommatidia from each other. (b) Electron micrograph of a transverse section of a mosaic eye, carrying wild-type ommatidia and crb mutant clones (asterisks) in the same eye. Loss of crb results in photoreceptor cells (PRCs) with defective shapes (compare Fig. 1c). (c) Electron micrograph of a transverse section of an ommatidia representing the seven PRCs (R1-R7), which are well separated by the interrhabdomeral space (IRS). (d) Higher magnification of a PRC, showing the rhabdomere, the adherens junctions (arrows), and the stalk membrane(s).

highly stereotype, trapezoid pattern. The outer PRCs Rl-R6 are located in the periphery, while the R7 and R8 cells occupy the distal and the proximal portion of the center, respectively. Therefore, each cross section shows only seven PRCs. In contrast to many other insects, such as the honeybee, Drosophila has an open rhabdom, in which the rhabdomeres of the PRCs are well separated from each other by the interrhabdomeral space (Fig. 2c). The diameter of the rhabdomeres of the central R7 and R8 is $\sim 1 \mu \mathrm{m}$, whereas that of the peripheral rhabdomeres Rl-R6 measures about $2 \mu \mathrm{m}$ (ref. 1). The microvilli of the rhabdomeres of the outer RI-R6 cells are slightly tilted towards one side in the distal portion of the retina and towards the other side in the proximal portion (2). This feature, termed rhabdomere twisting, decreases the sensitivity of the PRCs to polarized light. 


\subsection{Various \\ Phenotypes \\ Associated with \\ Distinct Mutations}

The rhabdomeres are functionally equivalent to the outer segments of vertebrate PRCs, as both contain the components of the phototransduction machinery. Rl-R6 contain Rhodopsin l (Rhl) as visual pigment, which is encoded by the ninaE gene $(3,4)$. R7 expresses either $\operatorname{Rh} 3$ or $\operatorname{Rh} 4(5,6)$, responsible for ultraviolet sensitivity (7), and R8 expresses either Rh5 or Rh6, which have blue and green sensitivity, respectively (8).

The highly stereotypic organization of the cells within each ommatidium is essential for proper functioning of the eye. In the last decades, many genes have been characterized, mutations in which effect different aspects of this organization, such as the number of PRCs per ommatidium, their proper arrangement, their morphology and/or function, the shape of the rhabdomere, including the length and/or alignment of the microvilli, or changes in the length of stalk membrane. Some mutations are described below. Mutations that affect cell fate specification of PRCs result in the lack of individual PRCs or a change in their identity. For example, in sevenless (sev) or bride of sevenless (boss) mutant flies, the R7 cell is absent (9-12). In eyes lacking rough the identity of R2 and R5 is affected, which impairs the specification of R3 and R4 (13). Mutations in genes affecting the number or arrangement of PRCs are often manifested in a rough eye phenotype, such as in Ellipse, a dominant mutation in the EGF-receptor homologue (14), in loss-offunction mutations of canoe (15), in gain-of-function mutations of sevenless $\left(\operatorname{sev}^{S 11}\right)$ (16), or upon overexpression of the membranebound cytoplasmic domain of Crumbs (17) (Fig. 1b). The rougheye phenotype of some calphotin alleles is the result of misoriented rhabdomeres and PRC death (18). Mutations associated with genes like orthodenticle (otd) and PpH13 alter the shape of the rhabdomeres by changing the length/size of the microvilli, but keep photoreceptor number unaltered $(19,20)$. In hypomorphic alleles of the gene encoding Myosin Va or upon early expression of the membrane-bound cytoplasmic domain of Crumbs ectopic rhabdomeres form $(21,22)$. In rhabdomeres mutant for canoe and cofilin rhabdomeres are confined to only the distal third of the retina, similar as in PRCs lacking crumbs, stardust, or DPATJ, which additionally exhibit a reduction in stalk membrane length and undergo light-dependent retinal degeneration (23-29). Mutations in spacemaker/eyes shut prevent the separation of rhabdomeres, thus leading to a closed rhabdom without any interrhabdomeral space $(30,31)$. Finally, mutations leading to defective organelles like endosomes, multivesicular bodies, or lysosomes result in defective photoreceptors by affecting the transportation of pigments $(32,33)$. Other mutations affect the function of PRCs without changing their morphology. Such defects can be easily detected by measuring the electroretinogram (ERG). The ERG reflects the summed activity of all PRCs and a superimposed, 


\subsection{Various \\ Approaches to Study Drosophila Photoreceptors}

"evoked potential" from the first optic ganglion, the lamina. Mutants with altered ERGs can be classified according to these two components $(34)$ (reviewed in $(35,36))$.

Pioneering work of Benzer and his collaborators identified mutants in the visual system based on a modified phototactic behavior. This phototactic behavioral test is the simplest way for detecting visual system impairment $(37,38)$. Shortly later, Heisenberg employed an optomotor response approach to study photoreceptor function (39) (reviewed in (40)). In the same year, a novel screening approach was developed, called deep pseudopupil (DPP), which allows to rapidly uncover defects in ommatidial and PRC morphology. DPP is an optical phenomenon based on the highly stereotypic arrangement of PRCs and the superposition of the virtual images of several rhabdomeres (41) (Fig. la, inset). Any gross disruption that affects retinal organization and hence the stereotypic arrangement of the rhabdomeres causes a loss of DPP, e.g., in $r d g C$ mutants (42). A further development of this method, called optical neutralization of the cornea, uses either the autofluorescent of rhodopsin or the fluorescence induced upon illumination of eyes expressing green fluorescent protein (GFP) from transgenes under the control of PRC-specific promoters. This assay allows to easily screen large numbers of flies and provides additional information, such as defects in morphogenesis, planar polarity, or cell death $(43,44)$ (Fig. lc, d). Once a defect has been detected using either DPP or optical neutralization, a more detailed analysis of the mutant phenotype has to follow, such as histological analysis under light and electron microscope, which provides information on the fine structure of the PRCs, such as the length of microvilli or stalk membranes, the presence of pigment granules and various other organelles, or the integrity of the base of the rhabdomere or the adherens junction. Eyes with morphological defects can be further analyzed by immunohistochemistry to search for defects in the localization of marker proteins. This can be achieved by either cryo-sectioning/labeling or whole mount labeling. Table 1 lists several antibodies, which label different compartments of adult PRCs. Mutants having structural defects and/or impaired protein localization can be further analyzed with electrophysiological methods for their visual response (36).

The genetic tools available in Drosophila research have allowed studying the function of many genes required for development and function of the eye in great detail. The genome sequences revealed not only that many genes are conserved between flies and human, but in addition that mutations in homologous genes can cause similar diseases/phenotypes in human and Drosophila. Strikingly, nearly $75 \%$ of human disease-causing genes are believed to have a functional homolog in flies (45). Therefore, and due to the fact that the 


\section{Table 1}

Some useful markers to label different compartments of adult PRCs

\begin{tabular}{|c|c|c|c|}
\hline Protein & Localization & Antibody & References $^{a}$ \\
\hline F-actin & Rhabdomere & Phalloidin ${ }^{\mathrm{b}}$ & \\
\hline Crumbs & Stalk membrane & Cq4 ${ }^{\mathrm{c}}$ (mouse, monoclonal) & $(27,69,70)$ \\
\hline Stardust & Stalk membrane & $\begin{array}{l}\text { Anti-Sdt }{ }^{\text {pep } 153} \text { (rabbit) } \\
\text { Anti-C-terminus } \\
\text { Anti-PDZ (rabbit and mouse } \\
\text { monoclonal mAB B8-1) } \\
\text { Anti-N-terminus (rat) }\end{array}$ & $\begin{array}{l}(72) \\
(73) \\
(26,74) \\
(75)\end{array}$ \\
\hline DPATJ & Stalk membrane & $\begin{array}{l}\text { Anti-DPATJ } \\
\text { Anti-Dlt }{ }^{\mathrm{d}} \text { (mouse and rabbit) }\end{array}$ & $\begin{array}{l}(23) \\
(71)\end{array}$ \\
\hline DE-Cadherin & Adherens junction & DCAD2 (mouse monoclonalc) & $(76)$ \\
\hline$\beta$-Catenin/Armadillo & Adherens junction & Anti-Arm N2 7Al ${ }^{c}$ & $(77)$ \\
\hline $\begin{array}{l}\text { Unconventional } \\
\text { myosin, P-Moesin }\end{array}$ & Rhabdomere base & & $(78,79)$ \\
\hline $\begin{array}{l}\mathrm{Na}^{+}-\mathrm{K}^{+} \text {-ATPase } \\
\alpha \text {-subunit }\end{array}$ & $\begin{array}{l}\text { Baso-lateral } \\
\text { membrane }\end{array}$ & $\begin{array}{l}\alpha 5 \text { (mouse monoclonal, } \\
\text { raised against the } \\
\text { chicken protein) }^{c}\end{array}$ & $(80,81)$ \\
\hline Cut & Nucleus & $2 \mathrm{~B} 10$ (mouse monoclonal) $^{\mathrm{c}}$ & $(82)$ \\
\hline $\begin{array}{l}\text { Spacemaker/eyes } \\
\text { shut }\end{array}$ & $\begin{array}{l}\text { Interrhabdomeral } \\
\text { space }\end{array}$ & $\mathrm{mAb} 21 \mathrm{~A} \sigma^{\mathrm{c}}$ & $(31,83)$ \\
\hline Chaoptin & $\begin{array}{l}\text { Lateral sides of } \\
\text { rhabdomeres }\end{array}$ & $\mathrm{mAB} 24 \mathrm{~B} 10^{\mathrm{c}}$ & $(83)$ \\
\hline Carbohydrate epitopes & $\begin{array}{l}\text { Rhabdomere } \\
\text { outline }\end{array}$ & Anti-HRP (Dianova) & $\begin{array}{l}\text { M. Mishra } \\
\quad \text { (unpublished) }\end{array}$ \\
\hline
\end{tabular}

andicates the origin of the antibody and/or the first publication showing staining in the eye

'Phalloidin is a bicyclic peptide isolated from the mushroom Amanita phalloides that selectively binds F-actin. Several variants are available, conjugated to different fluorophores (e.g., FITC, Alexa dyes, etc.)

'Monoclonal antibodies can be obtained from DSHB (http://dshb.biology.uiowa.edu/)

${ }^{\mathrm{d} D P A T J}$ was initially supposed to be discs lost (dlt) (71), but was later correctly mapped (84)

eye is a nonessential organ in flies, the Drosophila eye has become an ideal model to study the function of human genes involved in neurodegeneration, even of those genes that do not have a Drosophila counterpart (see, for example, $(46,47)$ for Drosophila as a model for polyglutamine disorders). Using the various techniques established over the years, it is easy to address biological questions concerning the function of genes in the Drosophila eye. By extensive mutagenesis screens using chemicals, e.g., ethyl methanesulfonate, ionizing radiation, e.g., X-rays, or transposons (e.g., P-elements) (reviewed in (48)), many mutations affecting eye development and function have been collected $(35,49)$. Besides detecting novel genes, 
mutagenesis screens can be used to identify additional components of a given signaling pathway active in the Drosophila eye. This approach was first applied to identify mutations that enhance or suppress the phenotype induced by mutations in sevenless, which encodes a receptor tyrosine kinase required for the specification of the R7 cells. In this screen Son of sevenless (Sos) was uncovered, which acts downstream of Sevenless and encodes a Ras guanine nucleotide exchange factor (GEF) $(50,51)$. Since then, this kind of screen has been frequently used as an unbiased approach, for example to identify modifiers of Tau-induced neurodegeneration in a Drosophila model for Alzheimer's disease (52).

While initially only viable mutations with a function in the eye could be detected in genetic screens, the induction of genetic mosaics using mitotic recombination allowed the identification and characterization of homozygous mutant tissues/cells in otherwise heterozygous animals. Thereby, the analysis of the later function of a gene, whose mutations result in lethality when the whole animal is mutant, is possible (53-55). This method was also used in screens aimed to identify novel genes with functions in the eye (56). Initially, $\mathrm{X}$-ray irradiation was used to induce somatic recombination, but the number of clones achieved was very low. The development of the yeast FLP/FRT system tremendously increased the frequency of mosaicism. This system is based on the introduction of the yeast flip recombinase (FLP) gene and its target sequences FRT ( $f$ ip recombinase recognition targets) into the fly genome (57). Upon activation of the FLP recombinase by a tissue- and/or stage-specific promoter, homozygous mutant cells are induced (Fig. 2b), which, depending on the proliferation capacity, give rise to large areas of mutant tissues. The introduction of an additional, lethal mutation on the chromosome bearing the wild-type allele makes it possible to produce eyes that are almost completely composed of mutant tissues (58). One of the promoters used to activate FLP recombinase is the heat-shock promoter, which activates FLP randomly in all tissues upon raising the temperature for a short period of time to $37^{\circ} \mathrm{C}$. Alternatively, ey-FLP, in which expression of FLP is under the control of the eye-specific eyeless (ey) promoter, efficiently generates flies in which almost all eye cells are mutant, while the rest of the animal is wild type, and thus permits the performance of simple Fl screens, aimed to identify eye-specific phenotypes (59). The possibility to mark either the mutant or the wild-type cells by GFP is a further advantage of this assay (60). With this method it can be determined whether a given gene is required in those cells, where it is active (cell autonomous function), or whether it exerts its function onto neighboring cells (nonautonomous function), e.g., if it encodes a signaling molecule.

Methods described above provide possibilities to study phenotypes obtained upon loss of function of a gene of interest. A useful complement to these strategies is the GAL4-UAS system, designed to induce stage- and tissue-specific (over)expression of a gene of 
interest (61). It is a bipartite system, in which one transgenic line, the activator or driver line, expresses the yeast transcription factor Gal4 in a known temporal and/or spatial pattern. The second transgenic line, the responder or effector line, carries the gene of interest (often only its coding region, obtained by cloning a cDNA) under the control of upstream activating sequences (UAS), the target sites of Gal4. To activate gene expression, activator and effector lines are crossed. The resulting progenies carry both transgenes and express the gene of interest under the control of the desired regulatory sequence (62). Further refinement of this method was achieved by the combination of the UAS/GAL4 system with a temperaturesensitive variant of the GAL4 repressor GAL80, called the temporal and regional gene expression targeting (TARGET) technique, in which GAL80ts is expressed under the control of the ubiquitous tubulin $1 \alpha$ promotor (62). By a simple temperature shift, GAL80 becomes inactivated and allows GAL4 to activate the UAS-gene of interest $((63)$; reviewed in $(64))$.

To summarize, Drosophila photoreceptors are accessible in vivo to a variety of genetic and cell biological techniques, including mosaic analysis, overexpression studies, immunohistochemistry, transmission and scanning electron microscopy, and confocal microscopy. Development, morphology, and function of photoreceptors are relatively invariant from animal to animal and well studied. Since the eye is not an essential tissue and not required for an animal's viability and fertility, it is a great system to study genes involved in neurodegeneration, including human genes not encoded in the fly's genome. For example, the overexpression of human ataxin-1, a gene associated with Spinocerebellar ataxia type 1 (SCAl), in the fly retina causes neurodegeneration similar to that observed in humans expressing a disease-inducing version of the ataxin protein characterized by expansion of a polyglutamine (polyQ) tract. More interesting, screening for genes that modify this phenotype in the fly uncovered genes that are involved in transcriptional regulation, RNA processing, and cellular detoxification (65), and are thus possible candidates as targets for future therapy. This and other examples demonstrate that the Drosophila eye is an ideal model to study the function of human genes involved in neurodegeneration (reviewed in (66-68)).

\section{Materials}

\subsection{Cryofixation \\ Reagents and Equipment}

1. 4\% Paraformaldehyde in phosphate-buffered saline (PBS) (obtained by dilution of 16\% (EM grade; from Electron Microscopy Sciences (EMS)).

2. PBS pH 7.4 and PBST (PBS $+1 \%$ Tween20) at room temperature. 
3. 10 and 25\% Sucrose (AppliChem, Cat no: 57-5-1) solution, diluted in PBS.

4. $5 \%$ Normal horse serum (NHS) blocking solution (dilute $30 \%$ NHS (ABD Serotec, Co9SA) with PBT).

5. Tissue embedding media NEG50 ${ }^{\mathrm{TM}}$ (Thermo Scientific).

6. Vectashield mounting media (Vector lab).

7. Coverslips $(24 \times 50 \mathrm{~mm})$.

8. $1.5 \mathrm{ml}$ Eppendorf tubes.

9. Dry ice.

10. Embedment molds for embedment (or cap of $1.5 \mathrm{ml}$ Eppendorf tube).

11. $1 \mathrm{ml}$ transfer pipettes.

12. Razor blade, glass slide, and sample holder.

13. Light microscope and cryostat.

2.2. Fixation for Light and Electron

Microscopy
2.3. Sectioning and Staining of Samples for Light Microscopy
1. 50\% Glutaraldehyde (EMS \#16320) diluted to $2.5 \%$ with Phosphate Buffer (PB) (EMS \#11600-05) and 16\% paraformaldehyde (EMS \#15700) diluted to $2 \%$ with $\mathrm{PB}$ for primary fixation in a $1.5 \mathrm{ml}$ Eppendorf tube (see Note 1).

2. $2 \% \mathrm{OsO}_{4}$ for secondary fixation: $\mathrm{Mix} 2$ parts $4 \% \mathrm{OsO}_{4}(\mathrm{EMS}$ \#19150) with one part $2 \% \mathrm{KMnO}_{4}$ solution $\left(\mathrm{KMnO}_{4}\right.$ in distilled water) and one part distilled water.

3. Ethanol, absolute, and diluted to $50,70,90$, and $100 \%$ (see Note 2).

4. Propylene oxide (EMS \#20410) for dehydration.

5. Rocking platform for agitation.

6. EPON: Mix the components of the Epon 812 kit (EMS \#14120) in a fresh plastic beaker according to the supplier's instruction: EMbed $812(20 \mathrm{ml})$, DDSA $(16 \mathrm{ml}), \operatorname{MNA}(8 \mathrm{ml})$, DMP-30 $(0.66 \mathrm{ml})$. Mix it for $1 \mathrm{~h}$ with a magnetic stirrer at room temperature (see Note 3 ).

7. Embedding mold (EMS \#70907) to embed the sample in resin.

8. Maintain an oven at $60^{\circ} \mathrm{C}$ for polymerization.

1. Ultramicrotome, razor blade, and Diamond Histo Knife.

2. To prepare glass knifes, break the glass (EMS \#71012) with a knife maker. Melt wax, dip a small container called the boat (to collect sections on water) into it, and immediately press it against the edge of the glass knife to fix it to the boat (Fig. 3).

3. To collect sections, mount an eyelash at one end of a toothpick by means of nail polish. 


\subsection{Sectioning and Staining of Samples for Electron Microscopy}

2.4.1. Coating of Grids

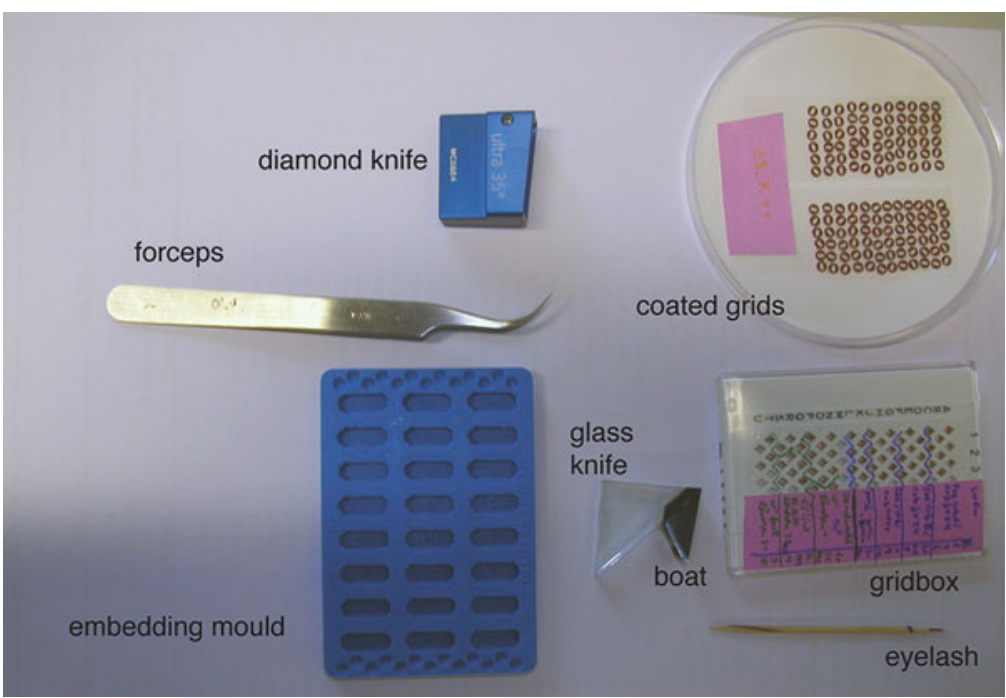

Fig. 3. Equipment required for the preparation of light and electron microscopy. See text for further explanation.

4. The toluidine blue solution $(0.25 \%$ Toluidine blue (EMS $\# 14950$ ) and $0.25 \%$ Sodium borate (EMS \#21130)) is prepared and mixed on a magnetic stirrer for $30 \mathrm{~min}$. Filter the solution and store it at room temperature (see Notes 4 and 5).

5. DPX (Sigma \#44581) for mounting the slide.

1. Wash single-slot grids (EMS \#G200F1-Cu) with acetone and let them dry.

2. For making formvar solution, dilute formvar (EMS \#15810) in $100 \% \mathrm{CHCl}_{3}$, to make a final concentration of $1 \%$.

3. Prepare glass slides by cleaning with a Kimwipe to remove fat and dust.

4. To coat the slides with a film of formvar, dip them into the formvar solution using the casting device (EMS \#71305-01) (Fig. 4a).

5. Take out the slides and let them dry inside the fume hood.

6. Cut out the film from the slide with a razor blade (Fig. 4b).

7. Prepare a rectangular cuvette filled with distilled water. Dip the slide with the film still attached to it into the water in an angle of $45^{\circ}$ (Fig. 4c). The film will detach from the slide and float on the water (Fig. 4d). A silvery appearance of the side of the film that was in contact with the slide indicates that it has the right thickness of 20-30 $\mathrm{nm}$.

8. Put the grids on the floating film with forceps so that the dull surface of the grid is oriented towards you (Fig. 4e). Once the 


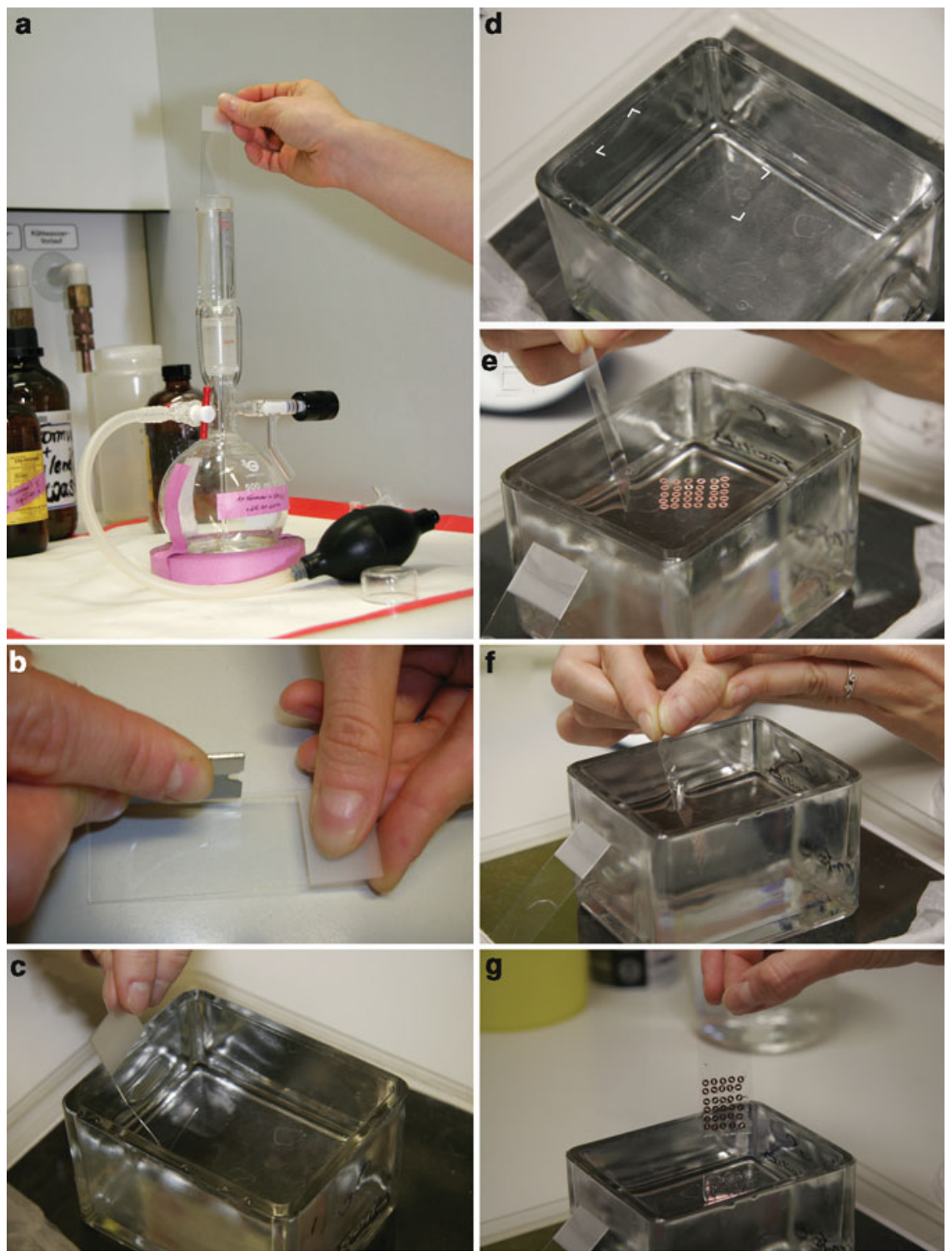

Fig. 4. Steps required for coating of grids. (a) Clean glass slide dipped inside the coating device containing the formvar solution. (b) Slide coated with formvar, cut by a razor blade to separate the film. (c) Slide with cut film dipped inside the water-containing cuvette to separate the film from the glass slide. (d) Grey film floating on top of the clean water (corners marked by white angles). (e) Grids are arranged on top of the thin film and the free end is used to pick up the grid along with the film. (f) Grids with the film are now sticking to the coverslip inside water. (g) Lifting of the grids from the water.

grids are attached to the formvar film, put a dust-free coverslip $(24 \times 50 \mathrm{~mm})$ on the free side of the film and press it against the water. This will lift the film with the grids from the water and attach them on the coverslip (Fig. 4f, g).

9. Put the coverslip on a petri dish with a filter paper (grids upside). After drying, grids are ready to be used. 
2.4.2. Sectioning and Staining of Grids

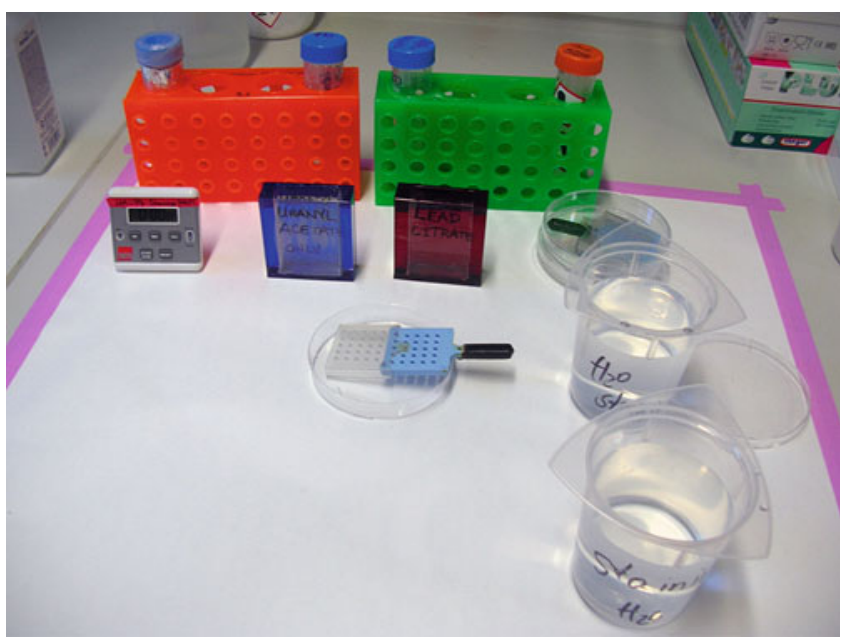

Fig. 5. Picture of grid-staining area showing the required tools. See text for further explanation.

1. Diamond knife, fine forceps, and filter paper cut into small triangular shape.

2. Lead citrate solution: Boil $500 \mathrm{ml}$ of water for $30 \mathrm{~min}$ (this will help to take out $\mathrm{CO}_{2}$ from water) and allow it to cool down to room temperature. Dissolve $0.2 \mathrm{~g}$ lead citrate (EMS \#27800) in $50 \mathrm{ml} \mathrm{CO}$-free water in a Falcon tube. Add $500 \mu \mathrm{l} 10 \mathrm{~N}$ sodium hydroxide. Shake the tube a bit. Let it stand overnight at room temperature to clear the solution. It is best when freshly prepared, but can be used up to 1 month stored at room temperature (see Note 6). Uranyl acetate (UA) solution: Dissolve UA (EMS \#22400) in 70\% methanol to make a final concentration of $2 \%$ and mix it for $\mathrm{l} \mathrm{h}$ in the dark. Fill the UA solution into a syringe and attach a filter (Millipore 0.22) at the tip. After filtering, the UA solution is ready for use. It is best when freshly prepared, but can be used up to 1 month when stored at $4^{\circ} \mathrm{C}$.

3. Grid-staining chamber (PELCO $\left.{ }^{\mathrm{TM}} 22510\right)$ and grid box (EMS \#71140) to store your samples with appropriate label (Fig. 5).

\section{Methods}

\subsection{Dissection of the Compound Eye}

1. Put the flies on a $\mathrm{CO}_{2}$ pad.

2. Cut off the head with a razor blade and pull out the proboscis with forceps without smashing the eyes.

3. The whole head can be used for cryo-, light-, and electron microscopy preparation. 
3.2. Methods to Score for Rhabdomere Phenotypes in the Intact Head

3.2.1. Deep Pseudopupil Analysis to Score for Defective Rhabdomeres

3.2.2. Optical

Neutralization

of the Cornea

3.3. Clonal Analysis
DPP analysis can be performed in living flies (41). Here it is described for isolated heads. Put the dissected heads with the "neck" on a slide. Shine blue light from the top (in the case of white-eyed flies) or white light from below (in the case of red-eyed flies) through the compound eye. The rhabdomeres efficiently absorb the light. Due to the precise architecture of the retina, the superposition of the pictures of several ommatidia appears as one single pseudopupil in the postero-dorsal quadrant of the eye (Fig. 1, inset). Any gross disruption of the regular retinal organization affects the stereotypic arrangement of rhabdomeres, and ultimately results in a loss of DPP $(41,42)$. The pseudopupil itself is best viewed using a low power $(10 \times)$ objective with a large optical aperture. Adjust the light source in order to maximize the contrast between the pseudopupil and the surrounding eye surface. Tilting the head can blur the image.

While the absence of a DPP demonstrates defects in the structure of the rhabdomeres, optical neutralization gives additional information on the nature of the defects. Dissected fly heads are mounted on a slide. If flies express a GFP-tagged protein, fluorescence can be used. Otherwise, one can make use of bright light microscopy for flies with pigmented eye.

Fluorescence microscopy using a water immersion objective can be performed with living flies $(43,44)$. Alternatively, heads are cut off from the flies and immersed in oil on a slide, covered with a coverslip, and viewed using an oil immersion objective $(63 \times)(\mathrm{N}$. Gurudev, personal communication).

Here we describe the clonal analysis of crumbs (crb) as an example of how to score a phenotype in the Drosophila eye of a mutant allele that leads to earlier lethality, using established methods essentially as described $(58,59)$. The mitotic recombination event is induced in heterozygous animals, where one chromosome carries a wildtype allele of $c r b$, and the other one a mutant allele. Upon mitotic recombination, followed by mitosis, two cell clones are formed, in which the cells are either homozygous for the wild-type allele or homozygous for the mutant allele. The sizes of the clones depend on the time point of induction, but also on the viability of mutant tissues. crb mutant clones will cover approximately $20-30 \%$ of the adult eye. They are scattered on the entire eye and can be recognized as white patches, since they are additionally marked by the recessive marker white $(w)$. To induce large eye clones, in which most of the eye is mutant for $c r b$, the FLP/FRT system is combined with a recessive lethal marker (e.g., lethal (3) cell lethal R3 (cl3R3) in the example below) so that those cells, which are homozygous for the wild-type allele, are at the same time homozygous for the lethal marker, thus undergoing apoptosis (60). Large crb mutant clones are obtained by setting up the following cross. 


\subsection{Light Microscopy Approach}

3.4.1. Tissue Fixation and Embedding for Light and Electron Microscopy

3.4.2. Dehydration and Embedding
P: yw P $\{e y$-FLP.N $\} / y w$ P $\{e y$-FLP.N $\} ; ; \mathrm{P}\{$ neoFRT $\} 82 \mathrm{~B} c l R 3$ $\mathrm{P}\left\{\right.$ white ${ }^{+}$un 1$\} 90 \mathrm{E} / T M 6 B \mathrm{X}$

$w / \Upsilon ; \mathrm{P}\{$ neoFRT $\} 82 \mathrm{~B} c r b^{11 A 22} / T M 6 B$

F1: yw P $\{e y-F L P . N\} / w ; P\{$ neoFRT $\} 82 \mathrm{~B} c l R 3 \mathrm{P}\left\{w h i t e^{+}-u n 1\right\} 90 \mathrm{E} /$ $\mathrm{P}\{$ neoFRT $\} 82 \mathrm{~B} c r b^{11 A 22}$

Eyes of adult Fl animals are mosaic, with the mutant clones being white and the few and small wild-type clones being red (Fig. 2b).

1. Fix the heads in an Eppendorf tube with 2\% paraformaldehyde and $2.5 \%$ glutaraldehyde in $\mathrm{PB}(\mathrm{pH} 7.2)$ at $4^{\circ} \mathrm{C}$ overnight as primary fixation (see Note 1).

2. Wash the heads thoroughly with PBS three times for $10 \mathrm{~min}$ each wash (solvent exchange).

3. The heads are subjected to secondary fixative for $2 \mathrm{~h}$ at room temperature in darkness with the secondary fixative $(0.2 \%$ OsO4 and $1 \% \mathrm{KMnO}_{4}$ in $\mathrm{PB}$ ). This step can be eliminated if the fixation is only for light microscopy.

4. Wash the heads with distilled water three times for $10 \mathrm{~min}$ each wash.

1. Dehydration is performed with a series of ethanol solutions $(50,70,90,95,2 \times 100 \%$ for 10 min each), followed by two washes with Propylene oxide (absolute) for $10 \mathrm{~min}$ each. Each step of dehydration should be carried out in a closed tube with shaking. After these steps heads are ready for infiltration with resin (see Note 2).

2. Infiltrate Epon mixture into the head with resin and propylene oxide according to Table 2 . All steps must be performed at room temperature with shaking under the fume hood (see Note 3).

3 . Embed the heads in pure resin in an embedding mold. You can add a small label with the genotype written on it into the mold. Fill the mold with pure resin and put the head in the mold

\section{Table 2}

\section{Infiltration with resin and propylene oxide}

\begin{tabular}{lllll} 
Steps & Propylene oxide: Epon & Ratio & Duration & Options \\
\hline 1. & Propylene oxide: Epon resin & $3: 1$ & $2 \mathrm{~h}$ & Cap closed \\
\hline 2. & Propylene oxide: Epon resin & $1: 1$ & Overnight & Cap closed \\
\hline 3. & Propylene oxide: Epon resin & $1: 3$ & $3 \mathrm{~h}$ & Cap closed \\
\hline 4. & Pure epon & & $3 \mathrm{~h}$ & Cap open \\
\hline
\end{tabular}


3.4.3. Sectioning and Staining of the Eye for Light Microscopy

\subsection{Electron}

Microscopy Approach perpendicular to the edge of the mold so that one eye is next to the edge. Keep the mold in the oven at $60^{\circ} \mathrm{C}$ and allow the block to polymerize for 12-24 h (see Note 7).

1. Trim the block to take out extra resin from the top of the eye if there is any. Trim the side of the block containing the specimen into a trapezoid and start collecting sections of $500 \mathrm{~nm}$ thickness. Trim $\sim 16 \mu \mathrm{m}$ from the cornea to reach the rhabdom.

2. Pick up the sections with a toothpick and collect them on a water droplet put onto a slide. Allow the droplet to dry on top of a hot plate (temperature $60-65^{\circ} \mathrm{C}$ ). This will help to stick the section properly on the slide. After the sections are dry put a little droplet of Toluidine blue solution to the sections. Allow it to stay on the hot plate for a few minutes, depending on the temperature of the plate, allowing the stain to dry. Wash the sections with distilled water and dry them over a hot plate. The rhabdom shape can be visualized with a $20 \times$ or a $40 \times$ objective under a light microscope (see Note 4). For the comparison of rhabdom size of several genotypes, it is necessary to take sections from the same depth of the rhabdom. The rhabdomere size diminishes and the interrhabdomeral space increases from the distal to the proximal part of the rhabdom. It is better to check for the rhabdom phenotype always at the level of the photoreceptor nuclei. The same block can be trimmed and used to check for the rhabdom phenotype under the electron microscope.

3. Another block should be used for the analysis of the phenotype in longitudinal sections. Take a perfect longitudinal section so that you can see the cornea, cone, rhabdom column, and basal lamina clearly under the light microscope (see Fig. 2a).

4. After sectioning and staining, add DPX to the slide and mount the specimens with a coverslip. For the rhabdom phenotype, take images under $63 \times$ or $100 \times$ magnification under an oil immersion objective. Pictures from the same magnification can be compared with the wild type to check for any mutant phenotype.

1. Flies having defective rhabdomeres as revealed by light microscopy can also be subjected for analysis at high resolution under the electron microscope. For this, the plastic block needs to be cut into $70 \mathrm{~nm}$ sections by means of a diamond/glass knife. Trim the block closer to the eye with a new razor blade in a trapezoid form, which will allow to get a "ribbon" of serial sections easily.

2. Collect the sections on formvar-coated single-slot grids. Ultrathin sections $(<70 \mathrm{~nm})$ should look grey or silver due to their reflection in water. Collect only those sections, which show a homogenous reflection on a silver surface. Align the sections 
3.6. Cryofixation and Immunolabeling of Eye Sections for Confocal Microscopy with an eyelash. Dip the grids into the water of the knife boat with forceps to collect the sections and collect the sections on the grid with the eyelash. Soak excess water with a filter paper, cut in a triangular shape. Store the grids in a grid box with appropriate label.

3. Put the sections on the grid into the grid-staining holder and slide the cover (Fig. 5). They are now ready for staining with uranyl acetate and lead citrate to give contrast under the electron microscope. Uranyl acetate stains the negative background like cytoplasm and lead stains membranous structures.

4. Put the grid-staining holder into a staining vessel. Fill the staining vessel with $70 \%$ methanol in order to keep the grids wet. Exchange the $70 \%$ methanol with UA solution in the staining box and keep for at least $10 \mathrm{~min}$. Wash the grids three times with 70\% methanol (solvent exchange) in the staining vessel. Thereafter, wash it three times in $50 \%$ methanol followed by three times in $30 \%$ methanol and finally three times in distilled water.

5. To give better contrast the grids are counterstained with lead citrate. Add lead citrate solution to the staining vessel and allow it to incubate for $5 \mathrm{~min}$. Wash three times with distilled water inside the chamber followed by three more washes inside the beaker filled with water. Allow the grids inside the grid chamber to dry. Transfer the grids into the grid box with appropriate label. Grids are ready for examination under the electron microscope.

6. To get an overall idea about the phenotype it is necessary to take images covering at least 6-8 ommatidia. In red-eyed flies, ommatidia are well separated from each other by pigment cells, which are filled with pigment granules (Fig. 2a, c). In whiteeyed flies, the outlining of the ommatidia can still be recognized despite the lack of pigments. The lower magnification picture will give a first impression about the rhabdom phenotype. The next step is to zoom in and take picture of individual ommatidia, which will allow, for example, to measure the length of microvilli and stalk membranes, to detect defective junctions or changes in the size or shape of organelles.

1. Place the fly heads in an Eppendorf tube with 4\% PFA for $30 \mathrm{~min}$ at room temperature in shaking condition. Wash the heads three times with PBS for 15 min each time.

2. Infiltrate the heads with $10 \%$ sucrose in PBS for $2 \mathrm{~h}$ on a rocker in a cold room. Transfer the heads to $25 \%$ sucrose solution and incubate them overnight in a cold room with gentle rocking.

3. Transfer the heads to the embedment mold filled with mounting media. Align the heads under a microscope in such a way that the eyes are looking towards the base of the mold/downwards. 
3.6.1. Cryosectioning

3.6.2. Cryolabeling
Put at least 12 heads in each boat, arranged in two rows. Place the boat over dry ice and allow it to freeze. The blocks are ready for sectioning or can be stored at $-80^{\circ} \mathrm{C}$ at this point for future use.

1. The key instrumentation for cryosection is the cryostat, which is essentially a microtome inside a freezer. Take the cryo sample holder and add a drop of mounting media to it. Take out the frozen block with forceps and stick it to the mounting media (see Note 8). Freeze the sample holder inside the cryostat until it is hard. Fix the sample holder to the cryostat and the eyes are ready for sectioning.

2. Trim the block in the form of a trapezoid with a thick razor blade. Adjust the cryotome to section at a thickness of $10-15 \mu \mathrm{m}$. Adjust the temperature of the cryostat to $-21^{\circ} \mathrm{C}$ for the blade and $-18^{\circ} \mathrm{C}$ for the object temperature (there should be always a temperature difference of $2-3^{\circ}$ between the object and the blade). Align the glass spacer and the blade properly so that there is a distance of $0.5 \mathrm{~mm}$ between the glass blade and the spacer; the spacer should be always ahead of the blade (see Note 9). Sections are picked up with a glass slide and stored inside the cryotome until you are done with the sectioning.

1. Wash the slides with PBS twice followed by one wash with PBST and incubate for $10 \mathrm{~min}$ at room temperature.

2. Block the slides with $5 \%$ NHS diluted in PBST for $1 \mathrm{~h}$ at room temperature. Add the primary antibody diluted with blocking solution. Keep it overnight at $4^{\circ} \mathrm{C}$ in a moist chamber to avoid drying.

3. Wash the slide three times with PBST, 10 min each wash. Add the secondary antibody diluted with blocking solution and keep the slide inside a wet chamber for at least $2 \mathrm{~h}$ at room temperature in the dark. At this step it is wise to add fluorescently labeled phalloidin along with the secondary antibody. Phalloidin binds to F-actin and provides a good staining for the overall shape and organization of the PRCs, in particular the rhabdomere.

4. Wash the slide with PBS, followed by two more washes with distilled water. Mount the slide with Vectashield with a coverslip and seal it with nail polish.

5. Slides are ready for examination under the confocal microscope. Take first an overview picture covering 6-8 rhabdoms. Then zoom in and adjust for one rhabdom to view the labeling more clearly. 


\subsection{Whole Mount Staining for Confocal Microscopy}

Whole mount staining allows to study photoreceptors without cryosectioning. This is a good method to study the localization of proteins at various developmental stages.

1. Dissect the eyes (take out the brain and the lamina) and place them in an Eppendorf tube containing 4\% PFA. Fix the eyes for $30 \mathrm{~min}$ at room temperature in shaking conditions.

2. Wash the eyes three times with PBS (20 min each wash).

3. Block the sample for $1 \mathrm{~h}$ with $5 \%$ NHS diluted with PBST.

4. Incubate with the antibody (diluted with blocking solution) by shaking overnight at $4^{\circ} \mathrm{C}$. If the antibody dilution has been used successfully on cryosections, then the antibody should work for whole mount staining with the same concentration.

5. Wash the eyes with PBST three times (20 min each wash) at room temperature. Add the appropriate secondary antibody along with phalloidin (this will allow you to see the rhabdom) and incubate at room temperature for $2 \mathrm{~h}$ in the dark.

6. Wash the eyes with PBS for $10 \mathrm{~min}$ followed by two more washes with water. Eyes are ready for mounting.

7. Remove the cornea with a needle. Make a bridge on the slide (by spacers) and add $100 \mu \mathrm{l}$ of Vectashield. Align the eyes under a dissecting microscope so that they face downwards. Add a coverslip. Mounting of the eye is a critical point. If properly done, z-stacks collected under the confocal microscope will allow to see every layer of photoreceptors.

\section{Notes}

Preparation of eye sections and their examination under the electron microscope is a lengthy process, where each and every step might cause poor quality or artifacts. The following steps help to reduce that.

1. Concentrations of glutaraldehyde and paraformaldehyde used for the primary fixation should be measured accurately. Higher fixative concentration dissolves the ultrastructure, resulting in poor-quality pictures.

2. Dehydration steps are very crucial and should be maintained as indicated. The tissue has to be properly and perfectly dehydrated, as resin is incompatible with water. Presence of water might induce holes in the resin-embedded blocks, making sectioning difficult.

3. Timing for infiltration steps has to be accurately maintained, because poor infiltration causes defective resin blocks and consequently difficulties in sectioning. In most red-eyed flies, the 
pigment granules seem to be washed away due to poor infiltration process. To avoid this, polymerization of the block should be allowed for more than $24 \mathrm{~h}$. If the problem persists, increase the time of step 3 (Table 1 ) to $12 \mathrm{~h}$.

4. Perfect staining of the sample is essential for examination of the rhabdom under the light microscope. Do not dry the stain completely (on the hot plate), which might result in overstaining of your sample. As a consequence, the rhabdom phenotype and/or the structure cannot be observed clearly. If this is the case, just wash your sample with $70 \%$ ethanol and stain it again.

5. If wrinkles are forming after staining increase the temperature of the hot plate.

6. Proper staining of specimens is essential for electron microscopy. Poor staining induces the formation of lead crystals, which spoil the quality of the image. To avoid lead crystal formation during staining, the first wash after lead citrate staining should be done rapidly. Few more washes should be carried out in order to remove lead citrate completely.

7. Chemicals used for fixation and staining for light/electron microscopy are highly toxic and should be handled under a fume hood. All materials in contact with the resin must be polymerized $\left(60^{\circ} \mathrm{C}, 24 \mathrm{~h}\right)$ before disposed to the regular waste.

8 . When cryosectioning, do not section the block immediately after taking it out from $-80^{\circ} \mathrm{C}$. Bring the block temperature to $-20^{\circ} \mathrm{C}$ before sectioning. This will result in smooth sectioning.

9. Look for the golden reflection with the blade and the glass spacer in the cryostat. A better alignment will help to get smooth sections.

\section{Acknowledgments}

We thank Michaela Rentsch for help with the electron micrographs, Franziska Friedrich for help in preparing Figs. 3, 4, and 5, and Nagananda Gurudev for the figure of the optical neutralization. Work of E. K. is supported by the Max-Planck Society (MPG) and a grant from the EC (HEALTH-F2-2008-200234).

\section{References}

1. Wolff T, Ready DF (1991) Cell death in normal and rough eye mutants of Drosophila. Development 113:825-839

2. Baumann O, Lutz K (2006) Photoreceptor morphogenesis in the Drosophila compound eye: Rl-R6 rhabdomeres become twisted just before eclosion. J Comp Neurol 498:68-79
3. Zuker CS, Cowman AF, Rubin GM (1985) Isolation and structure of a rhodopsin gene from D. melanogaster. Cell 40:851-858

4. O’Tousa JE, Baehr W, Martin RL, Hirsh J, Pak WL, Applebury ML (1985) The Drosophila ninaE gene encodes an opsin. Cell 40: $839-850$ 
5. Montell C, Jones K, Zuker C, Rubin G (1987) A second opsin gene expressed in the ultravioletsensitive R7 photoreceptor cells of Drosophila melanogaster. J Neurosci 7:1558-1566

6. Zuker CS, Montell C, Jones K, Laverty T, Rubin GM (1987) A rhodopsin gene expressed in photoreceptor cell R7 of the Drosophila eye: homologies with other signal-transducing molecules. J Neurosci 7:1550-1557

7. Feiler R, Bjornson R, Kirschfeld K, Mismer D, Rubin GM, Smith DP, Socolich M, Zuker CS (1992) Ectopic expression of ultraviolet-rhodopsins in the blue photoreceptor cells of Drosophila: visual physiology and photochemistry of transgenic animals. J Neurosci $12: 3862-3868$

8. Salcedo E, Huber A, Henrich S, Chadwell LV, Chou WH, Paulsen R, Britt SG (1999) Blueand green-absorbing visual pigments of Drosophila: ectopic expression and physiological characterization of the R8 photoreceptor cell-specific Rh5 and Rh6 rhodopsins. J Neurosci 19:10716-10726

9. Harris WA, Stark WS, Walker JA (1976) Genetic dissection of the photoreceptor system in the compound eye of Drosophila melanogaster. J Physiol 256:415-439

10. Reinke R, Krantz DE, Yen D, Zipursky SL (1988) Chaoptin, a cell surface glycoprotein required for Drosophila photoreceptor cell morphogenesis, contains a repeat motif found in yeast and human. Cell 52:291-301

11. Tomlinson A, Bowtell DD, Hafen E, Rubin GM (1987) Localization of the sevenless protein, a putative receptor for positional information, in the eye imaginal disc of Drosophila. Cell 51:143-150

12. Campos-Ortega JA, Jürgens G, Hofbauer A (1979) Cell clones and pattern formation: studies on sevenless, a mutant of Drosophila melanogaster. Wilhelm Roux's Arch Dev Biol 186:27-50

13. Tomlinson A, Kimmel BE, Rubin GM (1988) Rough, a Drosophila homeobox gene required in photoreceptors $\mathrm{R} 2$ and $\mathrm{R} 5$ for inductive interactions in the developing eye. Cell 55:771-784

14. Baker NE, Rubin GM (1989) Effect on eye development of dominant mutations in Drosophila homologue of the EGF receptor. Nature 340:150-153

15. Miyamoto H, Nihonmatsu I, Kondo S, Ueda R, Togashi S, Hirata K, Ikegami Y, Yamamoto D (1995) Canoe encodes a novel protein containing a GLGF/DHR motif and functions with Notch and scabrous in common developmental pathways in Drosophila. Genes Dev 9:612-625
16. Basler K, Christen B, Hafen E (1991) Ligandindependent activation of the sevenless receptor tyrosine kinase changes the fate of cells in the developing Drosophila eye. Cell 64:1069-1081

17. Grzeschik N, Knust E (2005) IrreC/rst-mediated cell sorting during Drosophila pupal eye development depends on proper localisation of $D E$ cadherin. Development 132:2035-2045

18. Yang Y, Ballinger D (1994) Mutations in calphotin, the gene encoding a Drosophila photoreceptor cell-specific calcium-binding protein, reveal roles in cellular morphogenesis and survival. Genetics 138:413-421

19. Mishra M, Oke A, Lebel C, McDonald EC, Plummer Z, Cook TA, Zelhof AC (2010) Pphl3 and orthodenticle define a dual regulatory pathway for photoreceptor cell morphogenesis and function. Development 137:2895-2904

20. Zelhof AC, Koundakjian E, Scully AL, Hardy RW, Pounds L (2003) Mutation of the photoreceptor specific homeodomain gene Pphl3 results in defects in phototransduction and rhabdomere morphogenesis. Development 130:4383-4392

21. Li BX, Satoh AK, Ready DF (2007) Myosin V, Rabll and dRipll direct apical secretion and cellular morphogenesis in Drosophila photoreceptor cells. J Cell Biol 177:659-669

22. Muschalik N, Knust E (2011) Increased levels of the cytoplasmic domain of Crumbs repolarise developing Drosophila photoreceptors. J Cell Sci 124:3715-3725

23. Richard M, Grawe F, Knust E (2006) DPATJ plays a role in retinal morphogenesis and protects against light-dependent degeneration of photoreceptor cells in the Drosophila eye. Dev Dyn 235:895-907

24. Johnson K, Grawe F, Grzeschik N, Knust E (2002) Drosophila Crumbs is required to inhibit light-induced photoreceptor degeneration. Curr Biol 12:1675-1680

25. Hong Y, Ackerman L, Jan LY, Jan Y-N (2003) Distinct roles of Bazooka and Stardust in the specification of Drosophila photoreceptor membrane architecture. Proc Natl Acad Sci U S A 100:12712-12717

26. Berger S, Bulgakova NA, Grawe F, Johnson K, Knust E (2007) Unravelling the genetic complexity of Drosophila stardust during photoreceptor morphogenesis and prevention of light-induced degeneration. Genetics 176: 2189-2200

27. Pellikka M, Tanentzapf G, Pinto M, Smith C, McGlade CJ, Ready DF, Tepass U (2002) Crumbs, the Drosophila homologue of human $\mathrm{CRBl} / \mathrm{RPl} 2$, is essential for photoreceptor morphogenesis. Nature 416:143-149 
28. Pham H, Yu H, Laski FA (2008) Cofilin/ADF is required for retinal elongation and morphogenesis of the Drosophila rhabdomere. Dev Biol 318:82-91

29. Matsuo T, Takahashi K, Suzuki E, Yamamoto D (1999) The Canoe protein is necessary in adherens junctions for development of ommatidial architecture in the Drosophila compound eye. Cell Tissue Res 298:397-404

30. Husain N, Pellikka M, Hong H, Klimentova T, Choe K-M, Clandinin TR, Tepass U (2006) The Agrin/perlecan-related protein eyes shut is essential for epithelial lumen formation in the Drosophila retina. Dev Cell 11:483-493

31. Zelhof AC, Hardy RW, Becker A, Zuker CS (2006) Transforming the architecture of compound eyes. Nature 443:696-699

32. Cheli VT, Daniels RW, Godoy R, Hoyle DJ, Kandachar V, Starcevic M, Martinez-Agosto JA, Poole S, DiAntonio A, Lloyd VK, Chang HC, Krantz DE, Dell'Angelica EC (2010) Genetic modifiers of abnormal organelle biogenesis in a Drosophila model of BLOC-1 deficiency. Hum Mol Genet 19:861-878

33. Pulipparacharuvil S, Akbar MA, Ray S, Sevrioukov EA, Haberman AS, Rohrer J, Kramer $\mathrm{H}$ (2005) Drosophila Vpsl6A is required for trafficking to lysosomes and biogenesis of pigment granules. J Cell Sci 118:3663-3673

34. Wu CF, Wong F (1977) Frequency characteristics in the visual system of Drosophila: genetic dissection of electroretinogram components. J Gen Physiol 69:705-724

35. Hardie RC,PostmaM(2008)Phototransduction in microvillar photoreceptors of Drosophila and other invertebrates. In: Basbaum AI, Kaneko A, Shephard GM, Westheimer G (eds) The senses: a comprehensive reference. Academic, San Diego, pp 77-130

36. Pak WL (2010) Why Drosophila to study phototransduction? J Neurogenet 24:55-66

37. Pak WL, Grossfield J, Whiten V (1969) Nonphototactic mutants in a study of vision of Drosophila. Nature 222:351-354

38. Hotta Y, Benzer S (1969) Abnormal electroretinograms in visual mutants of Drosophila. Nature 222:354-356

39. Heisenberg M (1971) Isolation of mutants lacking the optomotor response. Drosoph $\operatorname{lnf}$ Serv 112:65-93

40. Heisenberg M (1997) Genetic approach to neuroethology. Bioessays 19:1065-1073

41. Franceschini N (1972) Pupil and pseudopupil in the compound eye of Drosophila. In: Wehner R (ed) Information processing in the visual systems of arthropods. Springer, Berlin, pp 75-82
42. Steele F, O’Tousa JE (1990) Rhodopsin activation causes retinal degeneration in Drosophila rdgC mutant. Neuron 4:883-890

43. Pichaud F, Desplan C (2001) A new visualization approach for identifying mutations taht affect differentiation and organization of the Drosophila ommatidia. Development 128:815-826

44. Meyer NE, Joel-Almagor T, Frechter S, Minke B, Huber A (2006) Subcellular translocation of the eGFP-tagged TRPL channel in Drosophila photoreceptors requires activation of the phototransduction cascade. J Cell Sci 119:2592-2603

45. Pandey UB, Nichols CD (2011) Human disease models in Drosophila melanogaster and the role of the fly in therapeutic drug discovery. Pharmacol Rev 63:411-436

46. Whitworth AJ (2011) Drosophila models of Parkinson's disease. Adv Genet 73:1-50

47. Ambegaokar SS, Roy B, Jackson GR (2010) Neurodegenerative models in Drosophila: polyglutamine disorders, Parkinson disease, and amyotrophic lateral sclerosis. Neurobiol Dis 40:29-39

48. St. Johnston D (2002) The art and design of genetic screens: Drosophila melanogaster. Nat Rev Genet 31:176-188

49. Wang T, Montell C (2007) Phototransduction and retinal degeneration in Drosophila. Pflugers Arch 454:821-847

50. Rogge RD, Karlovich CA, Banerjee U (1991) Genetic dissection of a neurodevelopmental pathway: son of sevenless functions downstream of the sevenless and EGF receptor tyrosine kinases. Cell 64:39-48

51. Bonfini L, Karlovich CA, Dasgupta C, Banerjee U (1992) The son of sevenless gene product: a putative activator of Ras. Science 255:603-606

52. Shulman JM, Feany MB (2003) Genetic modifiers of tauopathy in Drosophila. Genetics 165:1233-1242

53. Garen SH, Kankel DR (1983) Golgi and genetic mosaic analyses of visual system mutants in Drosophila melanogaster. Dev Biol 96:445-466

54. Becker HJ (1957) Über Röntgenmosaikflecken und Defektmutationen am Auge von Drosophila und die Entwicklungsphysiologie des Auges. Z Indukt Abstamm Vererbungsl 88:333-373

55. Stern C (1936) Somatic crossing over and segregation in Drosophila melanogaster. Genetics 21:625-730

56. Thaker HM, Kankel DR (1992) Mosaic analysis gives an estimate of the extent of genomic involvement in the development of the visual system in Drosophila melanogaster. Genetics 131:883-894 
57. Golic KG, Lindquist S (1989) The FLP recombinase of yeast catalyzes site-specific recombination in the Drosophila genome. Cell 59:499-509

58. Stowers RS, Schwarz TL (1999) A genetic method for generating Drosophila eyes composed exclusively of mitotic clones of a single genotype. Genetics 152:1631-1639

59. Xu T, Rubin GM (1993) Analysis of genetic mosaics in developing and adult Drosophila tissues. Development 117:1223-1237

60. Newsome TP, Asling B, Dickson BJ (2000) Analysis of Drosophila photoreceptor axon guidance in eye-specific mosaics. Development 127:851-860

61. Brand AH, Perrimon N (1993) Targeted gene expression as a means of altering cell fates and generatingdominantphenotypes. Development 118:401-415

62. Elliott DA, Brand AH (2008) The GAL4 system: a versatile system for the expression of genes. Methods Mol Biol 420:79-95

63. McGuire SE, Le PT, Osborn AJ, Matsumoto K, Davis RL (2003) Spatiotemporal rescue of memory dysfunction in Drosophila. Science 302:1765-1768

64. McGuire SE, Deshazer M, Davis RL (2004) Gene expression systems in Drosophila: a synthesis of time and space. Trends Genet 20:384-391

65. Fernandez-Funez P, Nino-Rosales ML, de Gouyon B, She WC, Luchak JM, Martinez P, Turiegano E, Benito J, Capovilla M, Skinner PJ, McCall A, Canal I, Orr H, Zoghbi HY, Botas J (2000) Identification of genes that modify ataxin-1-induced neurodegeneration. Nature 408:101-106

66. Cook T, Zelhof A, Mishra M, Nie J (2011) 800 facets of retinal degeneration. Prog $\mathrm{Mol}$ Biol Transl Sci 100:331-368

67. Lu B (2009) Recent advances in using Drosophila to model neurodegenerative diseases. Apoptosis 14:1008-1020

68. Bonini NM, Fortini ME (2002) Applications of the Drosophila retina to human disease modeling. In: Moses K (ed) Drosophila eye development. Springer, Heidelberg, pp 257-271

69. Tepass U, Knust E (1993) Crumbs and stardust act in a genetic pathway that controls the organization of epithelia in Drosophila melanogaster. Dev Biol 159:311-326

70. Izaddoost $S$, Nam S-C, Bhat MA, Bellen HJ, Choi K-W (2002) Drosophila crumbs is a positional cue in photoreceptor adherens junctions and rhabdomeres. Nature 416:178-183

71. Bhat MA, Izaddoost S, Lu Y, Cho KO, Choi KW, Bellen HJ (1999) Discs lost, a novel
multi-PDZ domain protein, establishes and maintains epithelial polarity. Cell 96:833-845

72. Bachmann A, Schneider M, Grawe F, Theilenberg E, Knust E (2001) Drosophila Stardust is a partner of Crumbs in the control of epithelial cell polarity. Nature 414:638-643

73. Hong Y, Stronach B, Perrimon N, Jan LY, Jan YN (2001) Drosophila Stardust interacts with Crumbs to control polarity of epithelia but not neuroblasts. Nature 414:634-638

74. Bulgakova NA, Rentsch M, Knust E (2010) Antagonistic functions of Two stardust isoforms in Drosophila photoreceptor cells. Mol Biol Cell 21:3915-3925

75. Bulgakova NA, Kempkens Ö, Knust E (2008) Multiple domains of Drosophila Stardust differentially mediate localisation of the Crumbs / Stardust complex during photoreceptor development. J Cell Sci 121:2018-2026

76. Oda $H$, Uemura $T$, Harada $Y$, Iwai $Y$, Takeichi $M$ (1994) A Drosophila homolog of cadherin associated with armadillo and essential for embryonic cell-cell adhesion. Dev Biol 165:716-726

77. Riggleman B, Schedl P, Wieschaus E (1990) Spatial expression of the Drosophila segment polarity gene armadillo is posttranscriptionally regulated by wingless. Cell 63:549-560

78. Karagiosis SA, Ready DF (2004) Moesin contributes an essential structural role in Drosophila photoreceptor morphogenesis. Development 131:725-732

79. Satoh AK, Li BX, Xia H (2008) Calciumactivated myosin $\mathrm{V}$ closes the drosophila pupil. Curr Biol 18:951-955

80. Lebovitz RM, Takeyasu K, Fambrough DM (1989) Molecular characterization and expression of the $\left(\mathrm{Na}^{+}+\mathrm{K}^{+}\right)$-ATPase alpha-subunit in Drosophila melanogaster. EMBO J 8:193-201

81. Yasuhara JC, Baumann O, Takeyasu K (2000) Localization of $\mathrm{Na} / \mathrm{K}$-ATPase in developing and adult Drosophila melanogaster photoreceptors. Cell Tissue Res 300:239-249

82. Blochlinger K, Bodmer R, Jan LY, Jan YN (1990) Patterns of expression of cut, a protein required for external sensory organ development in wild-type and cut mutant Drosophila embryos. Genes Dev 4:1322-1331

83. Zipursky SL, Venkatesh TR, Teplow DB, Benzer S (1984) Neuronal development in the Drosophila retina: monoclonal antibodies as molecular probes. Cell 36:15-26

84. Pielage J, Stork T, Bunse I, Klämbt C (2003) The cell survival gene discs lost encodes a cytoplasmic Codanin-1 like protein, not a homolog of the tight junction PDZ-protein Patj. Dev Cell 5:841-851 